\title{
Managing FDG PET-positive sarcoidosis: "a riddle wrapped in a mystery inside an enigma"
}

\author{
To the Editor:
}

VORSELAARs et al. [1] have shown infliximab, a monoclonal antibody against tumour necrosis factor (TNF), to be an effective agent in the treatment of refractory sarcoidosis. We put forth some issues that are worth discussing among scientific societies.

The authors use the term "refractory FDG PET-positive sarcoidosis", although the term remains undefined in their study. There is no consensus definition of refractory sarcoidosis. We feel it is difficult to define such terminology for which many differential diagnoses are available. To guide clinicians in appropriate decision making, it is important that the criteria for selection of patients with refractory sarcoidosis are well defined.

Patients with high uptake of $18 \mathrm{~F}$-fluorodeoxyglucose (FDG) by positron emission tomography (PET) have been shown in tuberculosis (TB), sarcoidosis, and a wide array of inflammatory, infective and malignant pathologies. Sometimes TB and sarcoidosis may be difficult to differentiate clinically; however, FDG PET can help narrow down the differential diagnoses, detect extra-nodal involvement and be useful for post-therapy monitoring in such patients [2]. The authors concluded that those patients with refractory disease with a high standardised uptake value/uptake on the FDG PET scan are the ones most likely to benefit from infliximab therapy. However, advice to use FDG PET scans in patients with sarcoidosis may not be appropriate, as it can't be recommended routinely considering the cost and little additional benefit in patient management.

Looking at baseline characteristics, it is difficult to interpret the outcome from this study as 13 (23\%) patients had received some anti-TNF treatment before initiation of infliximab, and 24 (43\%) patients and $46(82 \%)$ patients were on concomitant corticosteroid and methotrexate treatment, respectively. These factors could have confounded the present outcome.

Anti-TNF therapies have provided effective treatment for a number of illnesses and it should be noted that they may be associated with an increased risk of reactivation of TB in patients with latent infection [3]. Along with blocking TNF-mediated immune responses, infliximab has been shown to interfere with innate immune responses, such as phagolysosome maturation and monocyte apoptosis, as well as cell-mediated responses, including interferon- $\gamma$ secretion by memory T-cells, complement-mediated lysis of Mycobacterium tuberculosis-reactive $\mathrm{CD}^{+} \mathrm{T}$-cells and increased regulatory T-cell activity [3].

Physicians should always be aware about the use of TNF- $\alpha$ blockers according to the guidelines. It is recommended to search for an active or latent TB before beginning treatment with infliximab, and to examine these patients frequently. Screening for TB infection should be used in patients along with possible prophylactic treatment during infliximab therapy [4].

Among the known infective agents, M. tuberculosis is one of the aetiological agents for sarcoidosis [5]. The circulating immune cells in sarcoidosis possess significant alterations in genome-wide gene expression supporting a "systemic" inflammatory nature of sarcoidosis that challenges current thinking about the compartmentalisation of inflammation in sarcoidosis. Significant concordance in gene expression patterns between sarcoidosis and TB have been demonstrated, which provide vital clues regarding sarcoidosis aetiology [6]. In their analysis MAERTZDORF et al. [7] reveal that the gene expression signatures in TB and sarcoidosis show very similar patterns, with a common up-regulation of proinflammatory pathways and interferon signalling, and close similarity to TB signatures. MicroRNA expression has also been shown to have a similar pattern in both conditions, whereas cytokines in the serum of TB patients revealed a slightly elevated proinflammatory pattern compared with sarcoidosis and controls [7].

The association is still "a riddle wrapped in a mystery inside an enigma" (quoted from Winston S. Churchill, October 1, 1939), and understanding of the dual aetiopathogenesis and their management needs further research before we reach a conclusion. especially in TB endemic areas http://ow.ly/U0de5 
Prasanta Raghab Mohapatra ${ }^{1}$, Kotyal Umamaheshwarappa Vivek $^{2}$, Manoj Kumar Panigrahi ${ }^{1}$ and Sourin Bhuniya ${ }^{1}$

${ }^{1}$ Dept of Pulmonary Medicine, All India Institute of Medical Sciences, Bhubaneswar, India. ${ }^{2}$ Government Medical College and Hospital, Chandigarh, India.

Correspondence: Prasanta Raghab Mohapatra, Dept of Pulmonary Medicine, All India Institute of Medical Sciences, Bhubaneswar 751019, India. E-mail: prmohapatra@hotmail.com

Received: July 162015 | Accepted: Aug 252015

Conflict of interest: None declared.

References

1 Vorselaars AD, Crommelin HA, Deneer VH, et al. Effectiveness of infliximab in refractory FDG PET-positive sarcoidosis. Eur Respir J 2015; 46: 175-185.

2 Karunanithi S, Kumar G, Sharma P, et al. Potential role of (18)F-2-fluoro-2-deoxy-glucose positron emission tomography/computed tomography imaging in patients presenting with generalized lymphadenopathy. Indian $J$ Nucl Med 2015; 30: 31-38.

3 Harris J, Keane J. How tumour necrosis factor blockers interfere with tuberculosis immunity. Clin Exp Immunol 2010; 161: 1-9.

4 Salmon-Ceron D. Recommendations for the prevention and management of tuberculosis in patients taking infliximab. Ann Med Intern (Paris) 2002; 153: 429-431.

5 Drake WP, Newman LS. Mycobacterial antigens may be important in sarcoidosis pathogenesis. Curr Opin Pulm Med 2006; 12: 359-363.

6 Koth LL, Solberg OD, Peng JC, et al. Sarcoidosis blood transcriptome reflects lung inflammation and overlaps with tuberculosis. Am J Respir Crit Care Med 2011; 184: 1153-1163.

7 Maertzdorf J, Weiner J 3rd, Mollenkopf HJ, et al. Common patterns and disease-related signatures in tuberculosis and sarcoidosis. Proc Natl Acad Sci USA 2012; 109: 7853-7858.

Eur Respir J 2016; 47: 346-347 | DOI: 10.1183/13993003.01154-2015 | Copyright @eERS 2016

From the authors:

We read with interest the correspondence from Mohapatra and colleagues regarding our paper "Effectiveness of infliximab in refractory FDG PET-positive sarcoidosis" [1]. We agree with Mohapatra and colleagues that the term refractory sarcoidosis is still under discussion. We defined refractory sarcoidosis in the methods section as "patients with severe sarcoidosis, unresponsive to first- and second-line treatment, or who have experienced severe side-effects from these agents". Diagnosis of sarcoidosis was made according to American Thoracic Society/European Respiratory Society criteria [2], including biopsy. These criteria also describe exclusion of other differential diagnoses. Using the described definition for refractory sarcoidosis yields a very heterogeneous group of patients, consisting of sarcoidosis patients that need third line immunosuppressive therapies, e.g. biologicals such as infliximab.

Mohapatra and colleagues also mention the costs of routinely performing 18F-fluorodeoxyglucose (FDG) positron emission tomography (PET) scans in sarcoidosis. Because of costs, but also radiation exposure, we do not recommend routine use of FDG PET scans in all patients with sarcoidosis. However, infliximab is a very expensive drug and our study does show additional benefit of performing an FDG PET scan prior to the start of treatment to predict treatment response [1]. This, in our opinion, justifies the use of FDG PET for individualised therapeutic decision-making in selected patients with severe sarcoidosis.

Furthermore, the authors point out the risk of tuberculosis in patients treated with infliximab. We agree that infliximab treatment is associated with an increased risk of reactivation of tuberculosis [3]. Therefore, all patients were screened for active or latent tuberculosis prior to treatment initiation, as noted in the exclusion criteria in the methods section [1]. Screening was performed by interferon- $\gamma$ release assay, and latent or active tuberculosis were not found in any of the patients. In the Netherlands tuberculosis is not endemic, however, in other parts of the world this problem might be of a different magnitude [4].

Lastly, the authors raise a very interesting question regarding the aetiology of sarcoidosis and mention Mycobacterium tuberculosis as one of the aetiological agents for sarcoidosis. Although the aetiology of sarcoidosis remains unresolved, we agree that mycobacterial antigens are an interesting candidate [5]. However, many other infectious causes, such as fungi, Borrelia, and Propionibacterium acnes have previously been shown to be associated with sarcoidosis [6-9]. Furthermore, non-infectious causes, such as inorganic substances and genetics, have also been implicated in the aetiology of this disease [6, 10]. As current treatment for sarcoidosis, including infliximab, is not considered curative and merely reduces inflammation, elusive insights into disease aetiology will probably lead to new, possibly curative therapies in the future [11]. 